\title{
Microalbuminuria: Marker or Maker of Cardiovascular Disease
}

\author{
Ahmed H. Abdelhafiz ${ }^{a}$ Shameela Ahmed ${ }^{a}$ Meguid El Nahas $^{b}$ \\ a Department of Elderly Medicine, Rotherham General Hospital, Rotherham, and bSheffield Kidney Institute, \\ Sheffield, UK
}

\section{Key Words}

Albuminuria - Vascular changes - Arterial sclerosis • Microalbuminuria · Glomerulosclerosis · Systemic endothelial dysfunction · Systemic atherosclerosis

\begin{abstract}
Advancing age is associated with albuminuria and vascular changes. This review will explore the putative links between the two. Vascular ageing involves endothelial dysfunction as well as increased arterial diameter, wall thickness and stiffness, ultimately leading to arterial sclerosis. This process is accelerated by a defective vascular repair process. Endothelial dysfunction is likely to be involved in the initiation and development of microalbuminuria. It is often followed by the development and progression of atherosclerosis. Initial$l y$, microalbuminuria is reversible but becomes fixed with the progression of vascular structural changes including glomerulosclerosis. The prevalence of microalbuminuria increases with age and has been shown to be a marker of widespread microvasculopathy at various levels including cerebral, cardiac and renal microcirculations. This has been linked to endpoint clinical events, with microalbuminuria increasing the risk of cognitive impairment and strokes, cardiovascular disease outcomes, and progression to end-stage renal
\end{abstract}

failure. Evidence of microvascular damage such as microalbuminuria associated with increased cardiovascular risk may suggest that microvascular damage and dysfunction predate overt macrovascular disease. Microalbuminuria and reduced glomerular filtration rate (GFR) may be markers of different pathologic processes. It is likely that microalbuminuria and reduced GFR simply represent, respectively, the spectrum of renal vascular manifestations from systemic endothelial dysfunction (microvascular disease) to systemic atherosclerosis (macrovascular disease).

Copyright $\odot 2011$ S. Karger AG, Basel

\section{Introduction}

The combination of large albumin molecular size and its negative charge accounts for its inability to pass into the urinary space; however, urinary albumin excretion up to $30 \mathrm{mg} /$ day is considered normal. The filtered albumin undergoes rapid endocytosis by proximal tubular cells. So microalbuminuria ( $>30-300 \mathrm{mg} /$ day) may result from a glomerular overfiltration or a tubular underreabsorption or a combination of both. Microalbuminuria is associated with salt sensitivity and hypertension, obesity, insulin resistance and diabetes as well as other compo-

\section{KARGER \\ Fax +41613061234 E-Mail karger@karger.ch} www.karger.com
Dr. Ahmed H. Abdelhafiz

Department of Elderly Medicine, Rotherham General Hospital

Moorgate Road

Rotherham S60 2UD (UK)

Tel. +44 01709307 576, E-Mail ahmedhafiz@hotmail.com 
nents of the metabolic syndrome such as dyslipidemia [1]. Microalbuminuria is also a feature of a number of inflammatory conditions including periodontitis, hepatitis and colitis. It can also be a response to both physiologic and abnormal stressors, such as exercise, posture, highaltitude hypoxia, and resolves once the stress is removed, suggesting a transient and fluctuating nature relating to renal vascular tone [1]. Microalbuminuria has been linked to abnormalities of the vasculature, such as endothelial dysfunction and reduced vascular dilatation [2]. The changes in urinary albumin excretion may therefore reflect underlying microvascular abnormalities. These can be triggered by a variety of hemodynamic, metabolic and inflammatory processes. Early in the course of these conditions, microvascular changes are reversible and the associated microalbuminuria is known to regress upon control of hypertension, obesity/diabetes and inflammation. Subsequently, the passage of albumin into the vessel wall would stimulate an inflammatory response, lipid accumulation, and eventually atherosclerosis. At this stage, the sclerotic changes affecting the renal microvasculature, i.e. glomerular capillaries, lead to fixed albuminuria and progressive loss of renal function. Dynamic microalbuminuria with fluctuating levels may precede fixed albuminuria with structural changes.

\section{Prevalence of Microalbuminuria in the Elderly}

Risk factors for microalbuminuria in the elderly are less well defined than in younger adults in whom hypertension and diabetes seem to be more strongly associated with microalbuminuria. The prevalence of microalbuminuria increases with age $(5.8,11.4$ and $22.7 \%$ among adults $20-49,50-69$ and $\geq 70$ years of age, respectively); however, the association of microalbuminuria with diabetes or hypertension is weaker in older people, suggesting that other factors may contribute to the high prevalence of microalbuminuria in older adults. These may include a number of putative factors associated with microalbuminuria including poverty and social deprivation, poor dental and oral hygiene, periodontitis as well as some components of the metabolic syndrome. Also, higher rates of exposure to nephrotoxic agents and medications in older people may be a factor. Another factor is atherosclerosis (as distinct from arteriosclerosis) which is more common among older adults and can cause direct renal damage and ischemia or reduced renal perfusion by causing heart failure from coronary-artery occlusive events. The high prevalence of microalbuminuria in the elderly could be related to pathological processes in the kidney. Histological changes like glomerulosclerosis, tubular atrophy, interstitial fibrosis, and arteriolar sclerosis are increasingly found with higher age even in the absence of hypertension and diabetes [5]. Microalbuminuria in the elderly shows a dynamic nature. In a community study of 1,378 Japanese nondiabetic individuals (mean age 63.9 years), microalbuminuria showed high variability (both progression and regression) and this variability was associated with older age, small changes in body weight, hemoglobin, and salt intake during 1 year of follow-up [6].

\section{Significance of Microalbuminuria}

Moderate reductions in estimated glomerular filtration rate (eGFR) are very common but of uncertain clinical significance in older people with about $50 \%$ of individuals over 70 years old meeting the current definition of chronic kidney disease (CKD). This has led to a controversy regarding the meaning and clinical usefulness of reduced GFR among the elderly [7]. It is not very clear whether the high prevalence of CKD among elderly persons is a consequence of normal physiologic aging or whether it represents a kidney disease [8]. Also the mortality relative risk associated with a given level of eGFR seems to be attenuated with age especially in those with mild to moderate reductions in eGFR [9]. Microalbuminuria independently increases the risk of mortality, myocardial infarction and progression to kidney failure at all levels of eGFR [10]. The association of microalbuminuria with mortality was also linear without threshold effect [11]. This suggests the need for incorporation of microalbuminuria in the future revisions of the classification system for CKD [10]. This is important as adding albuminuria to patients with CKD stages 3 to 4 substantially increases the predictive power to identify individuals at high risk for progression and death. A large proportion of patients with CKD stage 3 are at low risk for progression as long as their urine albumin concentration is low. At the same level of GFR, the risk rises substantially when albuminuria is present. This observation raises important questions about the association of CKD with cardiovascular disease (CVD), suggesting that part of the increased risk is caused by albuminuria, a marker of vascular dysfunction, rather than reduced GFR, a marker of renal dysfunction. Albuminuria is a marker/predictor of the rate of CKD progression, whereas GFR is a marker of how advanced the disease process is. In the early stages of 
CKD, increases in albumin excretion seem to be more important than small decreases in GFR [12, 13]. Microalbuminuria and low eGFR have been shown to be associated with adverse cardiovascular and renal outcomes independent of cardiovascular risk factors and of each other among 10,640 older patients aged around 66 years with type 2 diabetes [14]. O'Hare et al. [15] concluded that microalbuminuria may be a valuable tool for mortality risk stratification in 94,934 elderly individuals with diabetes mellitus and varying levels of eGFR. In patients over 75 years, microalbuminuria was independently associated with an increased risk of death at all levels of eGFR after adjusting for potential confounders, while in younger age groups this association was present at higher levels of eGFRs but seemed to be attenuated at lower levels. Low eGFR and microalbuminuria are synergistic cardiovascular mortality risk factors and age-stratified analyses showed that eGFR and microalbuminuria were particularly strong risk factors for persons over 70 years old. The combination of eGFR and albuminuria also improved the prediction of progression to end-stage renal disease even in the elderly [16]. The independent association of microalbuminuria and increased mortality [hazard ratio (HR) 1.73] has also been shown in a cohort of diabetic elderly individuals (mean age 78 years) followed up for 10 years [17]. In a study of 15,336 community-dwelling older people (median age 80 years) in the UK, there was a graded and independent increase in all-cause and cardiovascular mortality as GFR decreases especially in those with eGFR $<45 \mathrm{ml} / \mathrm{min} / 1.73 \mathrm{~m}^{2}$ after a median follow-up of 7.3 years [18]. Dipstick proteinuria was associated with all-cause but not cardiovascular mortality risk. Dipstick proteinuria did not improve stratification of risk of CVD mortality in older people, suggesting that in this age group eGFR alone captures most of the independent CVD mortality risk and the authors concluded that in the elderly, identification and management of CKD should focus on the select group with more severe reductions in eGFR. However, this study measured a single dipstick test, which is not a sensitive measure, and without quantification, it could not study whether there were graded responses across the range from microalbuminuria to heavy proteinuria. In another population-based prospective study of 16,958 relatively younger persons (mean age 52.5 years) without manifest vascular disease, the presence of CKD independently increased the risk of coronary heart disease (HR 1.55 for CKD stage 1, 1.72 for CKD stage 2, 1.39 for CKD stage 3a, 1.90 for CKD stage $3 \mathrm{~b}$, and 4.29 for CKD stage 4 after a median follow-up of 24 years). CKD increased both nonvascular mortality and vascular mor- tality, mainly from heart failure, cardiac arrhythmia, and pulmonary embolism. The presence of proteinuria independently increased the risk for coronary heart disease (HR 1.72), but the effect on mortality was not determined due to the small number of people who had proteinuria [19]. In the Rancho Bernardo study to examine the gender-specific cardiovascular and cardiac mortality risk of microalbuminuria in community-dwelling older adults (mean age 71 years at baseline) followed up for 8 years, microalbuminuria caused a two-fold increase in risk only in women, but this could be due to survival bias; as women develop cardiovascular disease at an older age than men, they would have been more likely to have survived to be in this cohort [20].

\section{Albuminuria and Brain Function}

Albuminuria, as a marker of endothelial dysfunction, may be associated with cerebrovascular disease and cognitive dysfunction. In a prospective study of 1,345 community-dwelling older adults (mean age 75 years), baseline albuminuria, but not eGFR, was associated with reduced cognitive function only in men after 6.6 years of follow-up. This may suggest that cognitive decline results from cerebral microvascular disease; however, the gender difference is not explained [21]. In another cross-sectional study doubling of albuminuria was associated with increased odds of dementia (OR, 1.12) compared with participants with normal cognition after adjustment for cardiovascular risk factors, suggesting shared susceptibility for microvascular disease in the brain and kidney in older adults [22]. Association of cerebral small-vessel disease and white-matter lesions with high serum cystatin C level has also been shown in a community-based Japanese study of 604 older people. Subjects with higher cystatin C levels accompanied with albuminuria had a greater risk for the presence of subclinical cerebral small-vessel disease than those with lower cystatin $\mathrm{C}$ levels without albuminuria [23]. Albuminuria was the kidney biomarker most strongly associated with risk of incident stroke in a longitudinal community cohort study of 3,287 participants without a history of stroke age $\geq 65$ years followed up for 10 years. The HR of any stroke was 2.10 , while the HR for eGFR was 1.29 and for raised cystatin C was 1.22. These findings suggest that albuminuria and reduced GFR are separate pathologic processes, with albuminuria conferring a risk of incident stroke independent of filtration rate [24]. The association of microalbuminuria and brain function has also been observed previously [25-30]. 
The extravasation of serum proteins as a result of brain microvascular disease (in parallel to albuminuria in nephrosclerosis) would account for the perivascular distribution of white matter hyperintensities on magnetic resonance imaging. This process occurs gradually but steadily and eventually leads to cognitive decline. The endothelial dysfunction-serum protein extravasation hypothesis addresses a possible way that microvascular disease could cause brain injury [31,32]. Evidence of microvascular damage such as microalbuminuria, retinopathy, or cerebral microvascular disease as indicated by the presence of white matter lesions on magnetic resonance imaging are associated with increased cardiovascular risk suggesting that microvascular disease predates macrovascular disease [33].

\section{Conclusion}

Age-associated microvascular changes are manifested by endothelial dysfunction and microalbuminuria. Macrovascular disease includes increased arterial diameter, wall thickness and stiffness, and ultimately atherosclerosis [30]. Endothelial dysfunction is an important early step in the process of clinical overt CVD. Impaired endothelium-dependent vasodilatation has also been associated with albuminuria [2]. These impairments in endo- thelial function are involved in the initiation and development of atherosclerosis. Both aging and end-stage renal disease are independently associated with exponential increases in mortality from CVD and exert an additive effect. GFR and albuminuria are poorly correlated, but they exert a synergistic effect on CVD outcomes [16]. Cardio-kidney-damage (C-K-D) has been suggested previously as a unifying concept to reflect the fact that albuminuria is a sign of systemic endothelial dysfunction affecting all vascular beds including the brain, eyes, heart and kidneys [30]. Microalbuminuria and reduced GFR may be markers of different pathologic processes. It is likely that microalbuminuria and reduced GFR simply represent the renal manifestations of systemic endothelial dysfunction (microvascular disease) and systemic atherosclerosis (macrovascular disease), respectively [31]. The pathophysiology of the independently increased risk associated with both low GFR and microalbuminuria as well as the risk factors for developing microalbuminuria and its differential impact on different age groups requires further exploration.

\section{Disclosure Statement}

The authors have no conflicts of interest to declare.

\section{References}

1 Danziger J: Importance of low-grade albuminuria. Mayo Clin Proc 2008;83:806-812.

-2 Malik AR, Sultan S, Turner ST, et al: Urinary albumin excretion is associated with impaired flow- and nitroglycerin-mediated brachial artery dilatation in hypertensive adults. J Hum Hypertens 2007;21:231-238.

-3 Knight SF, Quigley JE, Yuan J, et al: Endothelial dysfunction and the development of renal injury in spontaneously hypertensive rats fed a high-fat diet. Hypertension 2008; 51:352-359.

4 Islam TM, Fox CS, Mann D, et al: Age-related associations of hypertension and diabetes mellitus with chronic kidney disease. BMC Nephrol 2009;10:17.

5 Zhou XJ, Rakheja D, Yu X, et al: The aging kidney. Kidney Int 2008;74:710-720.

6 Suzuki K, Konta T, Takasaki S, et al: High variability of albuminuria in nondiabetic population: the Takahata Study. Clin Exp Nephrol 2009;13:452-459.
7 Glassock RJ, Winearls C: Screening for CKD with eGFR: doubts and dangers. Clin J Am Soc Nephrol 2008;3:1563-1568.

8 Abdelhafiz AH, Brown SHM, Bello A, El Nahas M: Chronic kidney disease in older people - physiology, pathology or both? Nephron Clin Pract 2010;116:c19-c23.

$\checkmark 9$ Raymond NT, Zehnder D, Smith SC, et al: Elevated relative mortality risk with mild-tomoderate chronic kidney disease decreases with age. Nephrol Dial Transplant 2007;22: 3214-3220.

10 Hemmelgarn BR, Manns BJ, Lloyd A, et al: Relation between kidney function, proteinuria, and adverse outcomes JAMA 2010;303: 423-429.

11 Chronic Kidney Disease Prognosis Consortium: Association of estimated glomerular filtration rate and albuminuria with allcause and cardiovascular mortality in general population cohorts: a collaborative meta-analysis. Lancet 2010;375:2073-2081.
12 Hallan SI, Ritz E, Lydersen S, et al: Combining GFR and albuminuria to classify CKD improves prediction of ESRD. J Am Soc Nephrol 2009;20:1069-1077.

13 Tonelli M, et al for the Alberta Kidney Disease Network: Using proteinuria and estimated glomerular filtration rate to classify risk in patients with chronic kidney disease. Ann Intern Med 2011;154:12-21.

14 Ninomiya T, Perkovic V, de Galan BE, et al: Albuminuria and kidney function independently predict cardiovascular and renal outcomes in diabetes. J Am Soc Nephrol 2009; 20:1813-1821.

15 O'Hare AM, Hailpern SM, Pavkov ME, et al: Prognostic implications of the urinary albumin to creatinine ratio in veterans of different ages with diabetes. Arch Intern Med 2010;170:930-936.

16 Hallan SI, Orth SR: The conundrum of chronic kidney disease classification and end-stage renal risk prediction in the elderly - what is the right approach? Nephron Clin Pract 2010;116:c307-c316. 
-17 Boer ID, Katz R, Cao JJ, et al: Cystatin C, albuminuria, and mortality among older adults with diabetes. Diabetes Care 2009;32: 1833-1838.

-18 Roderick PJ, Atkins RJ, Smeeth L, et al: CKD and mortality risk in older people: a community-based population study in the United Kingdom. Am J Kidney Dis 2009;53:950960.

19 Di Angelantonio E, Chowdhury R, Sarwar $\mathrm{N}$, et al: Chronic kidney disease and risk of major cardiovascular disease and non-vascular mortality: prospective population based cohort study BMJ 2010;341:c4986.

-20 Jassal SK, Langenberg C, von Mühlen D, et al: Usefulness of microalbuminuria versus the metabolic syndrome as a predictor of cardiovascular disease in women and men $>40$ years of age (from the Rancho Bernardo Study). Am J Cardiol 2008;101:1275-1280.

-21 Jassal SK, Kritz-Silverstein D, Barrett-Connor E: A prospective study of albuminuria and cognitive function in older adults: the Rancho Bernardo Study. Am J Epidemiol 2010;171:277-286.
2 Barzilay JI, Fitzpatrick Al, Luchsinger J, et al: Albuminuria and dementia in the elderly: a community study. Am J Kidney Dis 2008;52: 216-226.

23 Wadaa M, Nagasawaa H, Kawanami T, et al: Cystatin $\mathrm{C}$ as an index of cerebral small vessel disease: results of a cross-sectional study in community-based Japanese elderly. Eur J Neurol 2010;17:383-390.

24 Aguilar MI, O’Meara ES, Seliger S, et al: Al buminuria and the risk of incident stroke and stroke types in older adults. Neurology 2010;75:1343-1350.

25 Kuo HK, Lin LY, Yu YH: Microalbuminuria is a negative correlate for cognitive function in older adults with peripheral arterial disease: results from the US National Health and Nutrition Examination Survey 19992002. J Intern Med 2007;262:562-570.

26 Vupputuri S, Shoham DA, Hogan SL, et al: Microalbuminuria, peripheral artery disease, and cognitive function. Kidney Int 2008;73:341-346.
27 Abbatecola AM, Barbieri M, Rizzo MR, et al: Arterial stiffness and cognition in elderly persons with impaired glucose tolerance and microalbuminuria. J Gerontol A Biol Sci Med Sci 2008;63:991-996.

28 Barzilay JI, Fitzpatrick A L, Luchsinger J, et al: Albuminuria and dementia in the elderly: a community study. Am J Kidney Dis 2008; 52:216-226

29 Weiner DE, Bartolomei K, Scott T, et al: Albuminuria, cognitive functioning, and white matter hyperintensities in homebound elders. Am J Kidney Dis 2009;53:438-447.

30 Najjar SS, Scuteri A, Lakatta EG: Arterial aging: is it an immutable cardiovascular risk factor? Hypertension 2005;46:454-462.

731 El Nahas M: Cardio-kidney-damage: a unifying concept. Kidney Int 2010;78:14-18.

>32 David S: Knopman. Invited commentary: albuminuria and microvascular disease of the brain - a shared pathophysiology. Am J Epidemiol 2010;171:287-289.

>33 Strain WD, Elyas S, Gates PE, et al: Age-related change in endothelial and microvessel function and therapeutic consequences. Rev Clin Gerontol 2010;20:161-170. 